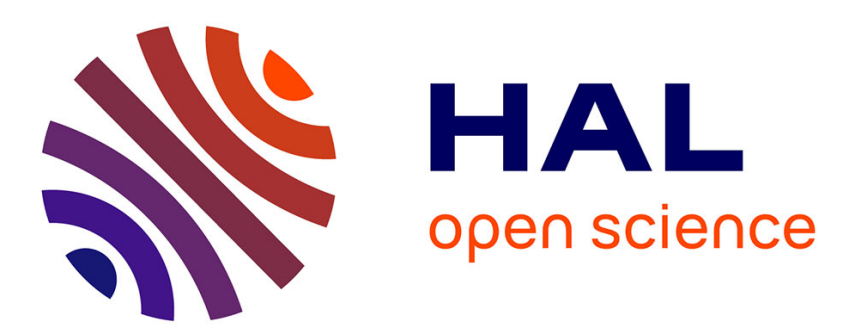

\title{
Towards X-ray medical imaging with robots in the open: safety without compromising performances
}

Lucas Joseph, Vincent Padois, Guillaume Morel

\section{To cite this version:}

Lucas Joseph, Vincent Padois, Guillaume Morel. Towards X-ray medical imaging with robots in the open: safety without compromising performances. International Conference on Robotics and Automation, May 2018, Brisbane, Australia. hal-01614508v2

\section{HAL Id: hal-01614508 \\ https://hal.science/hal-01614508v2}

Submitted on 23 Feb 2018

HAL is a multi-disciplinary open access archive for the deposit and dissemination of scientific research documents, whether they are published or not. The documents may come from teaching and research institutions in France or abroad, or from public or private research centers.
L'archive ouverte pluridisciplinaire HAL, est destinée au dépôt et à la diffusion de documents scientifiques de niveau recherche, publiés ou non, émanant des établissements d'enseignement et de recherche français ou étrangers, des laboratoires publics ou privés. 


\title{
Towards X-ray medical imaging with robots in the open: safety without compromising performances
}

\author{
Lucas Joseph ${ }^{1,2,3}$, Vincent Padois ${ }^{2}$ and Guillaume Morel $^{2,3}$
}

\begin{abstract}
In this paper, a control solution featuring an energetic constraint is developed to improve the safety of a robotic manipulator sharing its workspace with humans. This general control structure, exploits a generic safe controller that ensures the respect of multiple constraints thanks to a Linear Quadratic Problem formulation. With a unified energetic formulation, the controller allows to explicitly limit both the kinetic energy when moving and the wrench applied to the environment in case of contact with an unexpected obstacle. This control approach is experimented on a redundant Kuka LWR4+ robot which end-effector shall precisely point toward a given location while following a trajectory.
\end{abstract}

\section{INTRODUCTION}

$\mathrm{X}$-ray imaging is a technique widely used in different medical fields such as interventional procedures, cancer detection or radiology. An X-ray image is created through photons emitted from an X-ray source and collected by an imaging detector. This source can be still and provide a $2 \mathrm{D}$ representation of a targeted zone or it can move around the detector to obtain a 3D representation of the underlying body structure through filtered backprojection techniques [9].

When moving the source between two locations to build 3D images, a high speed is often desired. This is due to the fact that the duration of the imaging process shall be minimized while the patient and the targeted anatomical structure shall be immobile. Usually during the acquisition, the machine follows a preplanned trajectory that keeps the Xray source pointing toward the detector. However, the ability to adapt the trajectory of this source to account for human morphology, or to use already acquired images to plan online new positions of the X-ray source can improve the 3D volume reconstruction [20].

The rapid growth of collaborative robots opens new opportunities to perform such procedures with precise, redundant and torque sensing robots. The Cyberknife [2] is a first attempt to mount a radiation source on an industrial robot to perform radiotherapy. X-ray sources are also mounted on industrial robots to perform object analysis in industrial environments [4]. However, in these cases, the robot motion while shooting X-rays is slow and the surrounding environment is controlled. In the case of 3D imaging of a human body, the fast moving X-ray source poses some issues in terms of safety. In a constantly evolving environment such as an operating room, as exemplified in Figure 1,

\footnotetext{
${ }^{1}$ GE Healthcare, 78530 Buc, France lucas . joseph@ge.com

${ }^{2}$ Sorbonne Universités, UPMC Univ Paris 06, CNRS UMR 7222, Institut des Systèmes Intelligents et de Robotique, F-75005, Paris, France lastnamedisir.upme.fr

${ }^{3}$ INSERM, U1150, Agathe-ISIR, F-75005, Paris France
}

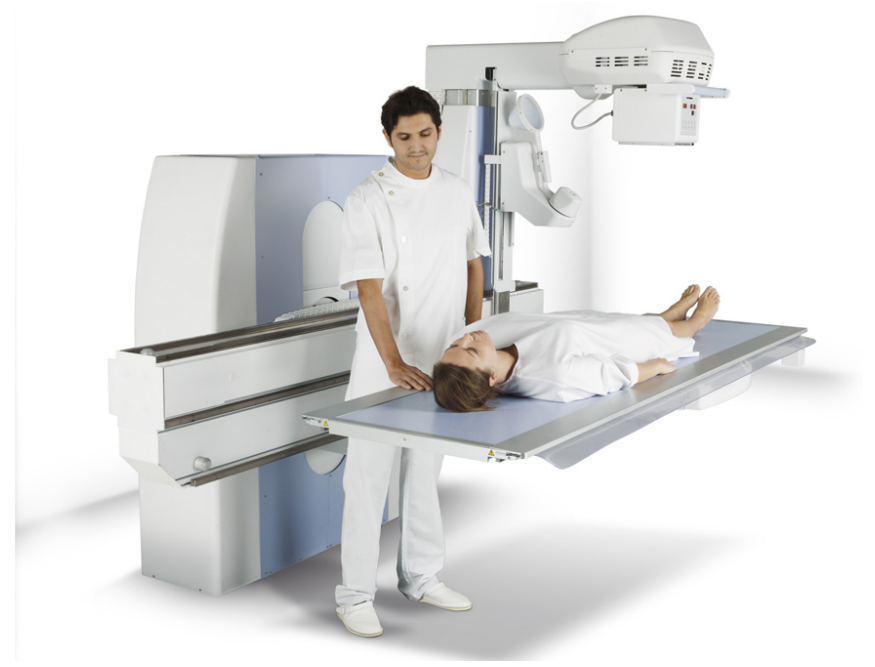

Fig. 1. Example of a radiographic situation with a robotic system carrying a radiation source near a patient and a radiologist. In this situation, unintended collision may arise between the robot and the environment and must be taken into account.

unwanted collision with humans (e.g. a patient) may occur. ISO Norm 15066 on collaborative robots [1] defines two types of undesired contacts: transient contact and quasi-static contact. In the first case the robot enters in contact with an obstacle that can recoil or retract. The ISO norm proposes in such case to restrain the robot kinetic energy that can be transferred from the robot to the human. The second case is representative of collision where the robot entraps an object with a fix environment. The ISO norm recommend in such case to limit the force/pressure applied by the robot to the obstacle.

In a known environment it is possible to plan safe trajectories with predefined velocities and torques to respect those safety norms. However, in the proposed context, ensuring safety cannot be done off-line as new control inputs must be computed reactively to account for the evolution of the environment or for a potential on-line adaptation of the trajectory.

In the considered scenario, the robot main task is to precisely point towards a target (i.e. the centre of the detector) while evolving in a shared space with humans who might unintentionally interact with the system. In case of undesired and unavoidable contact, safety measures must be enforced to protect the physical integrity of the colliding objects. Most importantly, the orientation of the source should always point towards the centre of the detector to avoid irradiating 
unwanted parts of the patient.

Impedance control is a tool of interest when dealing with interaction, but it requires a sophisticated tuning of the controller gains in order to be precise in free space and safe when in contact. It may require detecting a collision with algorithms such as the one proposed in [21] and to switch the controller gains between motions in free space and motion while in contact [10]. While for stability reasons, modifying the gain is not always desirable, it also has a direct impact on the quality of the trajectory tracking and on the pointing task. Furthermore, such control law may become unstable in case of a contact loss.

In this paper, a new safety criterion is proposed to handle both types of collision. By constraining the energy developed by the robot at each control time step, it is possible to limit both its kinetic energy during motion and its capacity to exert strong forces to reject disturbances due to unexpected contacts.

An important feature of the proposed approach arises from the lower level control framework. It is based on a formulation of the control problem as a convex optimization one. In this formulation, tasks are expressed as cost functions to be minimized according to a control variable, usually joint velocity or torque, and constraints are expressed as inequalities. This permits to dynamically account in an optimal and guaranteed way for the inequality constraints related to the physical limits of the robot [17]. It can also account for constraints arising from the surrounding environment [7], [16] such as the proposed safety criterion. If needed, hierarchies among tasks can also be accounted for in a strict way as in [12] or [18].

This work is an extension of the control approach proposed in [15] where two distinct energetic constraints are considered respectively for transient and quasi-static contact situations. In this work, both cases are handled using a single, reformulated energetic constraint. Furthermore, the proposed approach allows to indirectly limit the force that can be exerted by the robot in case of quasi-static contact. In a first section the control framework ensuring the correct positioning of the robot while pointing at a target and the respect of constraints is detailed. In a second section, the safety criterion chosen to ensure safety and its implementation are explained. The third section presents experimental results obtained on a 7 Dof robot. External measurement systems to record the correct realization of the pointing tasks are used to measure the efforts applied by the robot. The last section concludes on this works and mentions potential improvements.

\section{CONTROLLER DESCRIPTION}

The proposed context requires to have a robot that precisely points toward a target while following a trajectory in the 3D space. The robot must also strictly respect constraints imposed either by its intrinsic physical limits or by the environment. This section details the formulation of the different tasks and constraints and their implementation inside a quadratic solver.

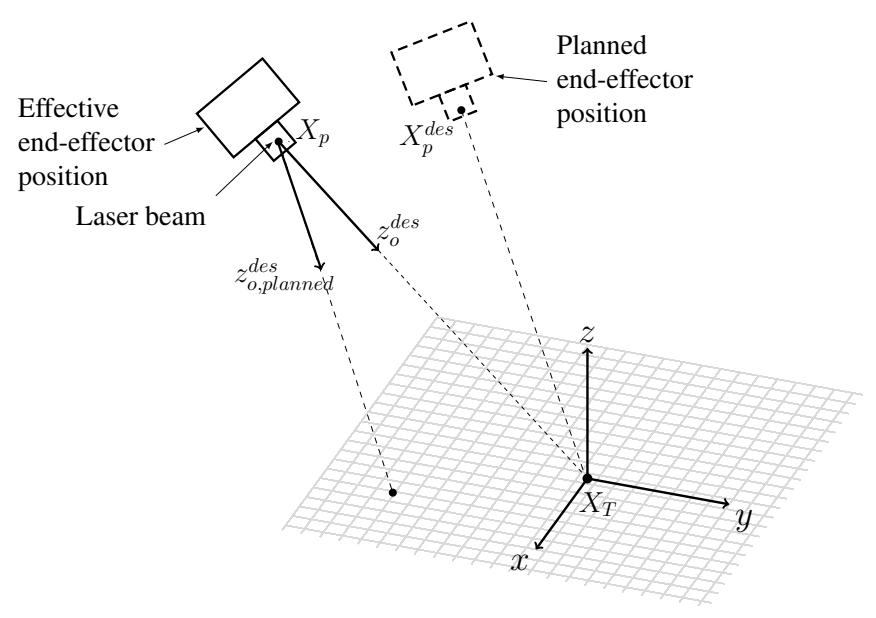

Fig. 2. When $X_{p}^{\text {des }}$ is not correctly tracked, $z_{o, p l a n n e d}^{\text {des }}$ is pointing at the wrong location. Hence, the orientation is computed on-line from the robot current location, $X_{p}$, to obtain the correct $z_{o}^{\text {des }}$.

\section{A. Tasks formulation}

To realize a 3D image of a targeted organ, the X-ray source needs to be moved around the patient. Hence, a trajectory tracking task is required. This trajectory can be computed off-line or in real-time and can be disturbed if an undesired contact occurs with the environment. Considering the application behind this control architecture, an orientation task is implemented to always keep the X-ray source pointing to the centre of the detector.

To the source is attached a frame $\left(P,\left(x_{o}, y_{o}, z_{o}\right)\right)$ with $z_{o}$ the main direction of the X-ray beam. The target point is $\mathrm{T}$, its position is $X_{T} \in \mathbb{R}^{3}$. $X_{p}$ is the current position of $P$ and $X_{p}^{\text {des }}$, its desired value, determined off-line.

Because $X_{p}$ can differ from $X_{p}^{\text {des }}$ (due to real time obstacle avoidance, for example), the desired value for $z_{0}$ is not computed off-line as $z_{o}^{\text {des }}=\frac{X_{T}-X_{p}^{\text {des }}}{\left\|X_{T}-X_{p}^{\text {des }}\right\|}$. Rather, it is computed on-line as: $z_{o}^{\text {des }}=\frac{X_{T}-X_{p}}{\left\|X_{T}-X_{p}\right\|}$.

The orientation error is then computed as the geodesic rotation from the current $z_{o}$ to the desired $z_{o}^{\text {des }}$.

\section{B. Control problem formulation}

The tasks to be performed are formulated in terms of controlled task space acceleration $\dot{\boldsymbol{v}}^{*} \in \mathbb{R}^{6}$, where $\boldsymbol{v} \in \mathbb{R}^{6}$ is the end-effector twist expressed at some point of interest. This acceleration can be decomposed into a linear part, $\dot{\boldsymbol{v}}_{p}^{*}$, and an angular part, $\dot{\boldsymbol{v}}_{o}^{*}$, which respectively represents the positioning and the pointing tasks. To ensure a precise positioning and pointing of the end-effector, the tracking of both tasks is ensured through the use of a PID + feed-forward regulators.

Overall, the position and orientation tasks $\boldsymbol{T} \in \mathbb{R}^{6}$ to be achieved can be written as a function to minimize of the general form

$$
\boldsymbol{T}=\dot{\boldsymbol{v}}^{*}-\dot{\boldsymbol{v}}
$$

with $\dot{\boldsymbol{v}}$ the model based operational acceleration, $\boldsymbol{v}=J(\boldsymbol{q}) \dot{\boldsymbol{q}}$, $J(\boldsymbol{q}) \in \mathbb{R}^{6 \times n}$ being the robot Jacobian, with $n$ the dimension 


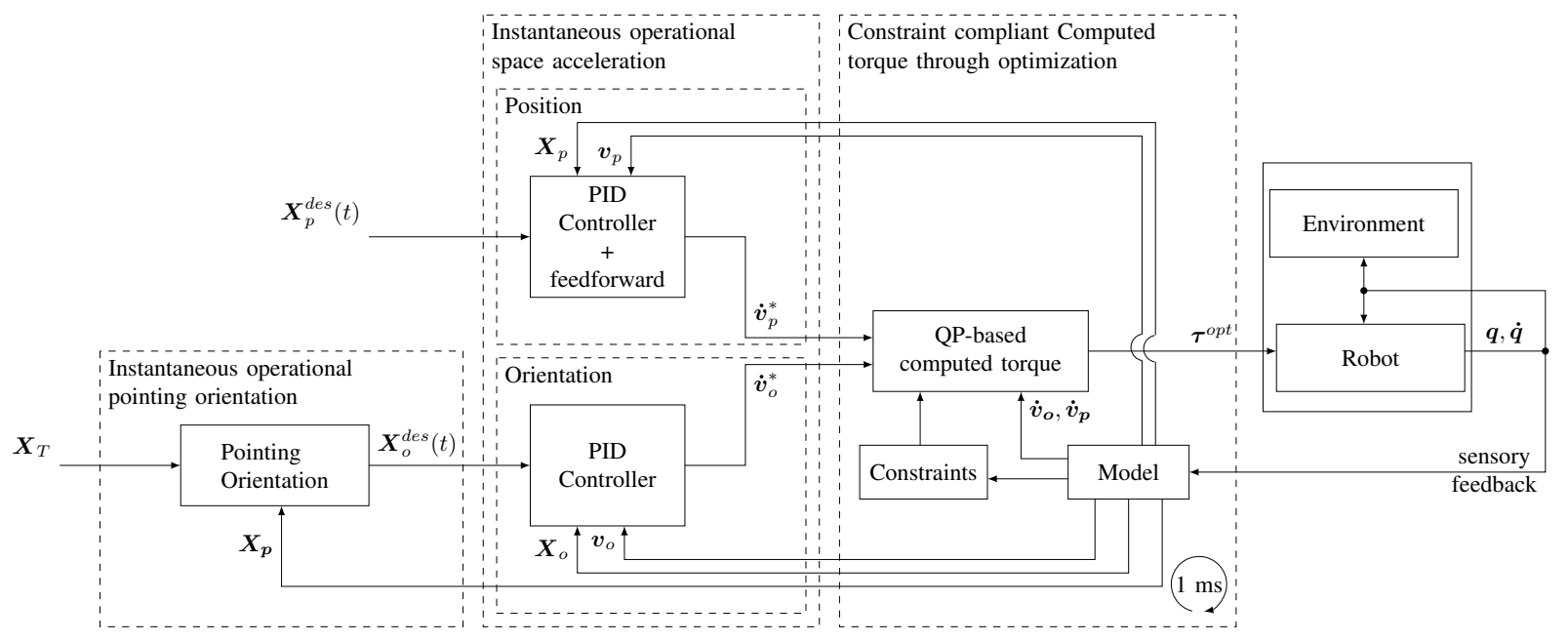

Fig. 3. Control scheme. The first block defines the desired position of the end-effector according to the trajectory and the desired orientation relatively to the robot current position. The second block computes the instantaneous operational space acceleration through a PID controller for both tasks $\dot{\boldsymbol{v}}_{\boldsymbol{p}}^{*}$ and $\dot{\boldsymbol{v}}_{\boldsymbol{O}}^{*}$. The third block computes the optimal torque command using a, constraint compliant QP-based, computed torque controller.

of the joint space, $\boldsymbol{q}$ and $\dot{\boldsymbol{q}} \in \mathbb{R}^{n}$ represent respectively the articular position and velocity vectors.

Considering a torque controlled robot, the acceleration tasks in (1) can be expressed as a function of joint torques, accounting for the equations of motion of the system

$\boldsymbol{T}(\boldsymbol{\tau})=\dot{\boldsymbol{v}}^{*}-\left(\dot{J}(\boldsymbol{q}) \dot{\boldsymbol{q}}+J(\boldsymbol{q}) M(\boldsymbol{q})^{-1}(\boldsymbol{\tau}-\boldsymbol{b}(\boldsymbol{q}, \dot{\boldsymbol{q}})-\boldsymbol{g}(\boldsymbol{q}))\right)$

where $\boldsymbol{b}(\boldsymbol{q}, \dot{\boldsymbol{q}}) \in \mathbb{R}^{n}$ is the centrifugal and Coriolis induced joint torque, $\boldsymbol{g}(\boldsymbol{q}) \in \mathbb{R}^{n}$ the gravity induced joint torque, $\boldsymbol{\tau} \in \mathbb{R}^{n}$ is the joint torque and $M(\boldsymbol{q})$ is the $n \times n$ jointspace inertia matrix.

Constraints: While tasks are a way to achieve desired behaviours with the robot, constraints are imposed to strictly avoid the violation of physical and actuation limits. The considered inequality constraints include the intrinsic constraints of the robot i.e. the bounds on joint positions, velocities and torques but also constraints imposed by the environment such as safety constraints. These inequalities can all be expressed as linear functions of $\tau$ using a proper discretization scheme such as in [17]. They can overall be written under the general form

$$
C(\boldsymbol{q}, \dot{\boldsymbol{q}}) \boldsymbol{\tau} \leq \boldsymbol{h}((\boldsymbol{q}, \dot{\boldsymbol{q}}))
$$

with $C(\boldsymbol{q}, \dot{\boldsymbol{q}})$ a constraint matrix and $\boldsymbol{h}(\boldsymbol{q}, \dot{\boldsymbol{q}})$ its associated limit vector.

Quadratic problem formulation: With the previous task description and constraint formulation, a Quadratic Problem $(\mathrm{QP})$ is defined as

$$
\begin{gathered}
\boldsymbol{\tau}^{\text {opt }}=\underset{\boldsymbol{\tau}}{\operatorname{argmin}} \frac{1}{2}\left(\|\boldsymbol{T}(\boldsymbol{\tau})\|^{2}+\varepsilon\|\boldsymbol{r}\|^{2}\right) \\
\text { s.t. (3) }
\end{gathered}
$$

where $\tau^{o p t}$ is the optimal torque, $\|$.$\| is the euclidean$ norm, $\boldsymbol{r}$ is a regularization term that ensures the uniqueness of the optimized solution and $\varepsilon \ll 1$ is a weighting term allowing to monitor the impact of the regularization on the main tasks performances.

For a kinematically redundant robot $(n>6)$ the choice of $\boldsymbol{r}$ is important as it affects the robot behaviour when the defined tasks do not fully constrain the robot. In the present work the controller is implemented on a redundant robot and the regularization task is set to minimize $\|\boldsymbol{\tau}-\boldsymbol{g}(\boldsymbol{q})\|^{2}$. This regularization function minimizes the difference between the computed torque and the gravity induced external torque.

Figure 3 depicts the whole control scheme. The first block on the left computes on-line the orientation necessary to keep the robot pointing toward the target knowing the measured position of the end-effector, $X_{p}$, and the position of the target $X_{T}$. The second block computes the instantaneous operational space acceleration through a PID controller. The last block contains the constraint compliant computed torque controller that takes as an input the desired acceleration and the constraints associated to the optimization scheme and computes the optimal torque to solve the problem.

\section{CONSIDERED DEFINITION OF SAFETY}

The previous section provides a control law that ensures a precise positioning of the robot and a constant pointing toward a target. However, accurate tracking requires a stiff PID tuning in order to efficiently reject disturbances. In the proposed context, this may lead to dramatically high interaction forces in case of unexpected contact.

In this section a safety constraint is formulated to handle both transient contact and quasi-static contact. In the first case, what is dangerous is the quantity of kinetic energy that can be transferred from the robot to the human. In the second case the quantity of force/pressure, resulting from the disturbance rejection behaviour of the PID controller when an unintentional contact with the environment (a human being in this case), needs to be monitored. 
Several contributions proposed control solutions to ensure safety in an unknown environment either by introducing mechanical compliance [5], by computing torque commands that limit the impact force with an obstacle [13] or by actively reacting in response to a collision detection [14]. However, they fail to consider both cases of collision or even to consider the level of dangerousness of the collision. It is desired to obtain a safety indicator, able to take into account both the robot kinetic energy during free motion, and its applied wrench in quasi-static contact.

This work proposes to use the work-energy theorem to link this two physical quantities. This theorem stipulates that the variation of kinetic energy from point $\mathrm{A}$ to point $\mathrm{B}$ is equal to the sum of the work of the forces exerted on the robot during the motion. It can be written

$$
\Delta E_{c}^{A \rightarrow B}=\int_{A}^{B} F d u(t)
$$

where $\Delta E_{c}$ is the variation of kinetic energy, $d u(t)$ defines the trajectory from point $\mathrm{A}$ to point $\mathrm{B}$ and $F$ is the equivalent actuation wrench required to follow this path.

This variation of kinetic energy can be used to quantify the dangerousness of a robot so that it does not generate too much kinetic energy while in free motion and does not apply too much efforts while in contact.

Given a robot, the kinetic energy of its effector at time $t_{k}$ can be written

$$
E_{c}\left(t_{k}\right)=\frac{1}{2} \boldsymbol{v}^{T}\left(t_{k}\right) \Lambda\left(\boldsymbol{q}\left(t_{k}\right)\right) \boldsymbol{v}\left(t_{k}\right) .
$$

where, dropping the time dependency for the sake of clarity, $\Lambda(\boldsymbol{q})=\left(J(\boldsymbol{q}) M^{-1}(\boldsymbol{q}) J^{T}(\boldsymbol{q})\right)^{-1} \in \mathbb{R}^{6 \times 6}$ is the operational-space inertia matrix. Given $\dot{\boldsymbol{v}}^{c}(t) \in \mathbb{R}^{3}$ the commanded acceleration trajectory, the velocity of the endeffector at time $t_{k}+T$ can be written

$$
\boldsymbol{v}\left(t_{k}+T\right)=\boldsymbol{v}\left(t_{k}\right)+\int_{t_{k}}^{t_{k}+T} \dot{\boldsymbol{v}}^{c}(t) d t .
$$

Considering a discrete time integration period $\Delta t$ such that $T=n \Delta t$ and $t_{k}=k \Delta t$, Equation (7) can be written in its discrete form

$$
\boldsymbol{v}_{k+n}=\boldsymbol{v}_{k}+\sum_{i=k}^{k+n-1} \dot{\boldsymbol{v}}_{i}^{c} \Delta t
$$

Considering this kinetic energy at time $t_{k}+T$ and assuming that the modification of the configuration of the robot between control instants $k$ and $k+n$ is small enough, the difference of kinetic energy between time $t_{k}+T$ and $t_{k}$ can be written

$$
\Delta E_{c}=\underbrace{\left(\boldsymbol{v}_{k} \Delta t+\frac{1}{2} \sum_{i=k}^{k+n-1} \dot{\boldsymbol{v}}_{i}^{c} \Delta t^{2}\right)^{T}}_{\Delta \boldsymbol{x}_{[k ; k+n]}} \underbrace{\boldsymbol{\Lambda}_{k} \sum_{i=k}^{k+n-1} \dot{\boldsymbol{v}}_{i}^{c}}_{\sum_{i=k}^{k+n-1} \boldsymbol{F}_{i}^{c}} .
$$

On the one hand, $\Delta \boldsymbol{x}_{[k ; k+n]}$ represents the expected variation of pose given an initial operational state $\left\{\boldsymbol{x}_{k} ; \boldsymbol{v}_{k}\right\}$ and the commanded acceleration trajectory $\dot{\boldsymbol{v}}_{[k ; k+n]}^{c}$ over the control window $[k ; k+n]$. On the other hand, $\sum_{i=k}^{k+n-1} \boldsymbol{F}_{i}^{c}$, where $\boldsymbol{F}_{i}^{c}=\Lambda_{k} \dot{\boldsymbol{v}}_{i}^{c}$, can be interpreted as the sum of the equivalent control wrenches over the control window $[k ; k+n]$.

This expression of the variation of kinetic energy is quadratic. While an optimization base computed-torque controller could rely on a method handling quadratic constraints, expressing the constraint in a linear form is preferable for real-time control purposes. One can notice that in the expression of $\Delta \boldsymbol{x}_{[k ; k+n]}, \dot{\boldsymbol{v}}^{c}$ is actually a desired control acceleration $\dot{\boldsymbol{v}}^{*}$ more than a control input in itself. This acceleration is known at instant $\mathrm{k}$ and can be used to compute the expected pose variation that would be induced if this acceleration was actually achieved. This leads to the modified expression of the kinetic energy at the next time step

$$
E_{c, k+1}=E_{c, k}+\left(\boldsymbol{v}_{k} \Delta t+\frac{1}{2} \dot{\boldsymbol{v}}_{k}^{*} \Delta t^{2}\right)^{T} \boldsymbol{\Lambda}_{k} \dot{\boldsymbol{v}}_{k}^{c}
$$

This formulation can be interpreted as the provisional kinetic energy in $\Delta t$ seconds. It can be expressed as a function of the actual control input: the joint torque $\tau_{k}$.

Using the forward velocity kinematic relation and the equation of motion, the operational space acceleration is written

$$
\dot{\boldsymbol{v}}_{k}=J_{k} M_{k}^{-1}\left(\boldsymbol{\tau}_{k}-\boldsymbol{b}_{k}-\boldsymbol{g}_{k}\right)+\dot{J}_{k} \boldsymbol{v}_{k} .
$$

Transient contact: The transient contact phase is directly handled by this constraint formulation. ISO Norm 15066 specifies acceptable energy limit, $E_{c}^{l i m}$, that can be transferred from the robot to different body parts. Substituting $\dot{\boldsymbol{v}}^{k}$ from (11) in (10) it is possible to constrain the robot maximum kinetic energy so that

$$
E_{c, k+1}(\tau) \leq E_{c}^{l i m}
$$

Quasi-static contact: During quasi-static contact, the robot is against an immobile obstacle. Thus, $\boldsymbol{v}_{k}=0 \mathrm{~m} / \mathrm{s}$ and $E_{c, k}=0 \mathrm{~J}$. The provisional kinetic energy (10) becomes

$$
E_{c, k+1}=\frac{1}{2}\left(\dot{\boldsymbol{v}}_{k}^{*} \Delta t^{2}\right)^{T} \boldsymbol{F}_{k}^{c}
$$

Equation (13) states that the efforts applied by the robot during quasi-static contact are a function of its kinetic energy and its commanded operational acceleration.

During contact the PID controller integrates the error to reject the perturbation. Therefore, $E_{c, k+1}$ will increase until reaching the constraint $E_{c}^{l i m}$. Using anti-windup techniques to saturate the output of the PID controller, the controlled operational acceleration $\dot{\boldsymbol{v}}_{k}^{*}$ also reaches a maximum $\dot{\boldsymbol{v}}_{k}^{*, s a t}$. This saturation being known, one can compute an integration period $\Delta t$ to indirectly limit the force $\boldsymbol{F}^{l i m}$ applied by the robot

Replacing each terms in (13), $\Delta t$ can be chosen so that 


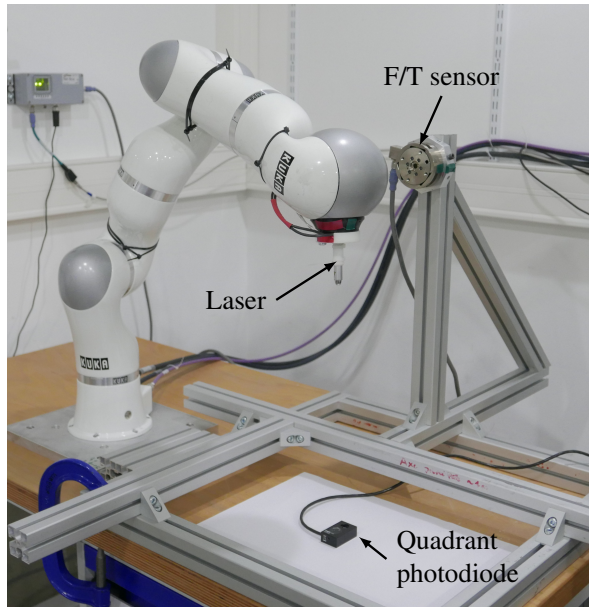

Fig. 4. Experimental setup with a KUKA robot, a laser, an ATI F/T sensor and a quadrant photodiode. The two measurement devices are only used for validation of the proposed control approach.

$$
\Delta t=\sqrt{\frac{2 E_{c}^{l i m}}{\left(\dot{\boldsymbol{v}}_{k}^{*, s a t}\right)^{T} \boldsymbol{F}^{l i m}}} .
$$

$F^{\text {lim }}$ can for example be chosen based on the maximum applicable efforts for different body parts recommended by ISO Norm 15066.

\section{EXPERIMENTAL RESULTS}

The control framework proposed in section II is implemented as a C++ OROCOS component [6] and tested on a KUKA LWR4+. This robot is a 7 DOF robot specially designed for human-robot interaction [3]. The KUKA Fast Research Interface is used to communicate with the robot at $1 \mathrm{kHz}$. The QP problem is implemented within qpOASES, a real-time open-source QP solver [8]. To measure the contact wrench applied by the robot an ATI F/T sensors is used. A laser is attached at the tool-tip of the robot and points toward a quadrant photodiode that records the position of the laser beam in the $x-y$ plane. This information is used to measure the precision of the pointing task. Similarly to the contact wrench measure, this information is not used by the controller, just for validation of the performances of the proposed control approach. The general setup is illustrated on Fig. 4. This section depicts several experiments realized to show the correct behaviour of the proposed control approach and to demonstrate its capacity to ensure safety in both cases of contact. In these experiments, the positioning error represents the distance between the desired position of the end-effector and its actual position estimated through the position of the encoders. The pointing error corresponds to the distance between the laser beam and the target.

During the experiments, the robot is performing a typical motion to obtain a 3D image through X-rays. To do so it moves along a line from $(0.5,-0.2,0.4) \mathrm{m}$ to $(0.5,0.2,0.4) \mathrm{m}$ from the robot base while pointing at a target located at $(0.5,0.0,0.013) \mathrm{m}$. A path is created with the KDL library [19] and computes a trapezoidal velocity profile with a

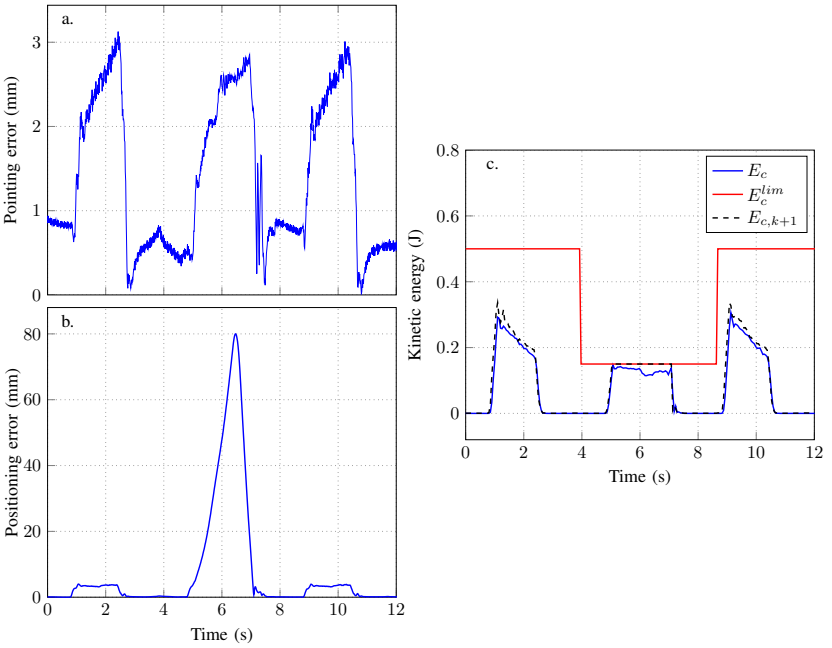

Fig. 5. Evolution of (a.) the pointing error, (b.) the positioning error, (c.) the current kinetic energy (blue line), $E_{c}^{l i m}$ (red), the provisional kinetic energy $E_{c, k+1}$ (dashed).

maximum velocity of $0.25 \mathrm{~m} / \mathrm{s}$ and a maximal acceleration of $1 \mathrm{~m} / \mathrm{s}^{2}$. The limit on kinetic energy is set at $0.5 \mathrm{~J}$ and a limit on force of $70 \mathrm{~N}$ is imposed. The PID controller gains are tuned with the Ziegler-Nichols method without any activated constraints and result in a stiffly actuated robot. The kinetic energy constraint integration period, $\Delta t$, is set at 16.5 ms using Equation (14). The experiments presented in this section can also be observed in the video attached to this paper.

\section{A. Kinetic energy constraint in free space}

This first experiment features a succession of motion of the robot along the defined trajectory for different value of $E_{c}^{l i m}$. The first motion is realized with a limit of $0.5 \mathrm{~J}$, sufficient to realize the positioning task. This is the nominal case. The second motion is realized with an energy limit of $0.15 \mathrm{~J}$ which is less than the one required to optimally follow the trajectory. The kinetic energy constraint of the robot, its current operational kinetic energy, $\frac{1}{2} \boldsymbol{v}^{T} \Lambda \boldsymbol{v}$, the positioning and the pointing error are recorded. Figure 5 depicts the results of this experiment.

When the limit on kinetic energy is higher than the one required to realize the desired motion, the mean positioning error is $2.7 \mathrm{~mm}$ and the average pointing error is $2.10 \mathrm{~mm}$. In steady state, the pointing error is below $1 \mathrm{~mm}$. When $E_{c}^{\text {lim }}$ is set to $0.15 \mathrm{~J}$, the available kinetic energy is insufficient to correctly follow the trajectory. Indeed, the torque computed by the QP solver must induce a provisional kinetic energy $E_{c, k+1}$ (dashed in Figure 5 c.) that does not go beyond $E_{c}^{\text {lim }}$. Consequently, the optimal solution requires a slower motion which induces a positioning error up to $80 \mathrm{~mm}$ from the planned trajectory. However, the expression of the orientation task as a function of the current robot position allows maintaining the pointing error similar to the one in the nominal conditions.

Overall the first part of this experiment shows that the robot is accurately following the trajectory and the pointing 


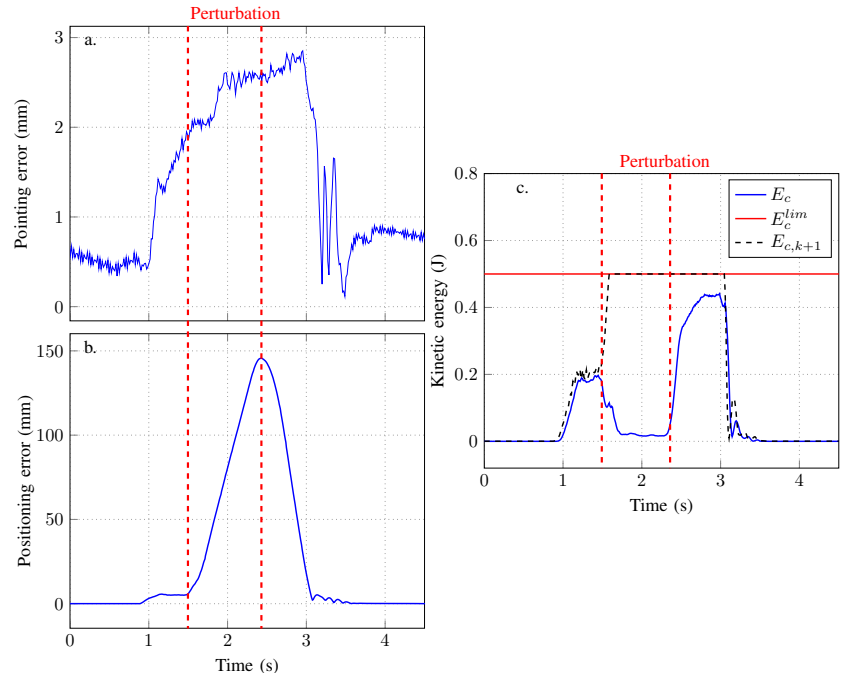

Fig. 6. Evolution of (a.) the pointing error, (b.) the positioning error, (c.) the current kinetic energy (blue line), $E_{c}^{l i m}$ (red), the provisional kinetic energy $E_{c, k+1}$ (dashed).

task is correctly performed with the formulated QP problem, including the energetic constraint. The second part shows that when insufficient energy is provided to the controller, the robot is still pointing at the target while the position error is rising which is an optimal solution to the problem from a safety point of view.

\section{B. Transient contact}

As explained in section III, during transient contact, what is important is the quantity of kinetic energy that will be transferred from the robot to the obstacle. The first experiment shows that it is possible to impose a maximum kinetic energy during the motion. However, when the contact occurs, a PID controller will tend to accumulate error, resulting in a sudden and dangerous release of energy when contact breaks. The proposed constraint on the variation of the kinetic energy should ensure that the robot energy never goes beyond a safety limits in such case. To assess this behaviour, a human operator is preventing the robot from moving for a brief period. Figure 6 depicts the results of this experiment.

It can be observed that when the robot is perturbed and almost stopped, the current kinetic energy becomes null while the provisional kinetic energy quickly reaches the defined limit. For the same reasons as stated before this induces a rise of the positioning error of up to $145 \mathrm{~mm}$. The interaction between the robot and the human also induces a pointing error of around $3 \mathrm{~mm}$. When the perturbation is released, the PID controller tries to impose a Cartesian acceleration that would induce a kinetic energy greater than the limit. That is why the provisional kinetic energy (dashed on figure 6 c.) stays at the limit: the QP solver computes a torque solution compatible with this constraint. The system then reduces its error until accomplishment of the trajectory.

This experiment shows the interesting characteristics of the proposed solution with a robotic behaviour that provides a
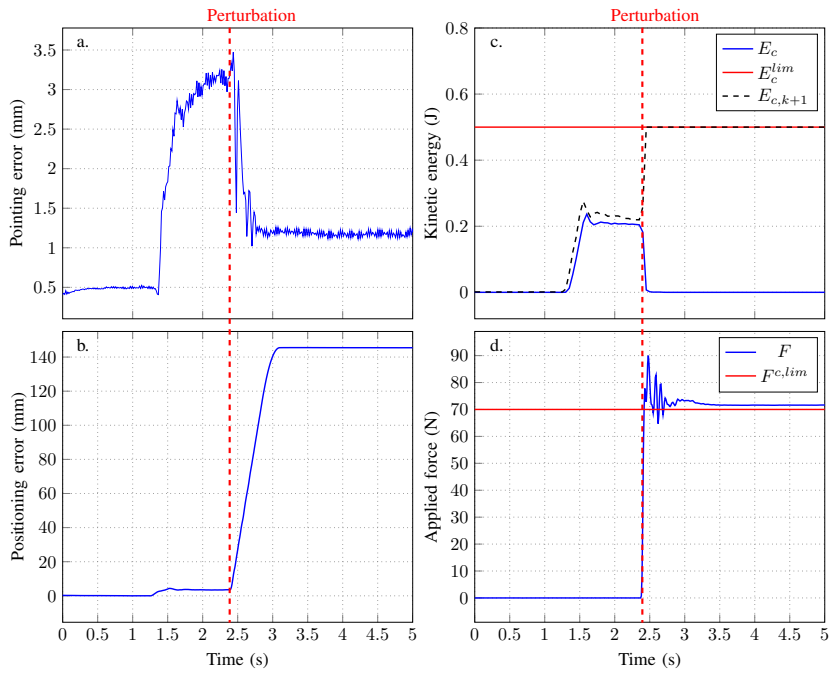

Fig. 7. Evolution of (a.) the pointing error, (b.) the positioning error, (c.) the current kinetic energy (blue line), $E_{c}^{l i m}$ (red), the provisional kinetic energy $E_{c, k+1}$ (dashed) and (d.) the contact wrench (blue) and its limit (red).

meaningful protection against sudden release of energy when contact breaks.

\section{Quasi-static contact}

When the robot hits a fix obstacle, the applied contact wrench must not exceed a limit specified by the ISO Norm 15066. To asses that the kinetic energy constraint can also be used to limit the contact wrench, an ATI sensor that records forces and torques, is mounted on a girder that is placed on the robot trajectory. Figure 7 shows the results of this experiment.

When contact occurs, a similar behaviour as the one in the previous experiment can be observed only this time the perturbation is constant and against a fixed obstacle. The force applied by the robot (Figure 7 d.) when contact is established is superior to the limited one but goes to acceptable limits for impact force during transient contact according to ISO Norm 15066. Once the controller energy reaches its limit and $\dot{\boldsymbol{v}}_{k}^{*}$ reaches its saturation, there is a stabilization of the applied efforts to values slightly above the limit (72 N). The observed overshoot is a consequence of the energy dissipated during the impact and is unavoidable, even if the amount of energy to be dissipated is monitored. The $2 \mathrm{~N}$ error that can be observed during steady state is due to the imperfect model of the robot and to the approximations made in the equations developed in section III. Consequently, the positioning error rises to important values of more than $140 \mathrm{~mm}$. Once again, during the perturbation, the pointing task is not affected by the constraint and keep being fulfilled.

During free motion the robot is stiffly actuated and precisely tracks the desired position. When contact occurs the limit on kinetic energy induces torque commands that prevent the robot from applying dangerous contact wrenches and results in a more compliant robot actuation. 


\section{CONCLUSION}

The controller presented in this paper constrains the variation of a robot kinetic energy in order to ensure safety and formally respect ISO norms for collaborative robots. This variation directly relates both to the energy that the robot could transfer when colliding an obstacle and to the contact wrench applied by the robot in case of a static contact. Since the energetic safety constraint is to be dynamically updated during the task execution, it is implemented as an explicit constraint within a QP-based computed torque controller, which guarantees to find the optimal solution without comprising safety. The experiments presented in section III demonstrate the application of the control scheme to a situation where the robot must point to a target at any time while performing a motion along a line to mimic a practical 3D X-ray imaging procedure.

Both the theoretical derivation and the experimental results allow pointing out a few remarkable features of the approach.

- The controller exploits PID control with gains that are tuned independently of the safety constrains. Namely, one may choose high gains to maximize the positioning precision; since the controller accounts for safety constraints at its lowest level, using high PID gains will never result in releasing high energy when unexpectedly colliding an obstacle.

- When a contact has been established with an unexpected obstacle, the wrench is limited to a maximal constant value, independent of the obstacle position. In other words, up to the controller bandwidth, the robot exhibits a null impedance and can be moved without adding extra force.

- The approach requires no more specific controller tuning than those of the PID compensators.

- This implementation of safety constraints does not require any collision detection algorithm nor any switching between different control modes.

- A natural property of the QP solver approach is to ensure constraints priority over tasks. A task requiring too much kinetic energy is degraded (the desired value is not followed anymore) while the other tasks continue to be correctly fulfilled. As a practical result, we have experimentally demonstrated that the pointing task towards the target was always fulfilled even when an obstacle prevented the robot from being able to bring the end-effector at its desired value.

For future work, several extensions are being considered. While the controller applies to a general context of safety, modelling of the environment and tool geometry is still to be provided in order to provide appropriate limit to respect [11]. Furthermore, although a medical procedure is targeted here, industrial applications can be considered as well, as in [16].

\section{ACKNOWLEDGMENT}

This work has been partially funded by ANRT under CIFRE grant $n^{\circ}$ 2015/0288.

\section{REFERENCES}

[1] ISO/TS 15066. Robots and robotic devices collaborative robots, 2016.

[2] JR Adler, SD Chang, MJ Murphy, J Doty, P Geis, and SL Hancock. The cyberknife: a frameless robotic system for radiosurgery. Stereotactic and functional neurosurgery, 69(1-4 Pt 2):124128, 1997.

[3] A. Albu-Schäffer, S. Haddadin, Ch. Ott, A. Stemmer, T. Wimböck, and G. Hirzinger. The DLR lightweight robot: design and control concepts for robots in human environments. Industrial Robot: An International Journal, 34(5):376-385, 2007.

[4] Hussein Banjak, Marius Costin, Caroline Vienne, and Valérie Kaftandjian. X-ray Computed Tomography Reconstruction on Non- Standard Trajectories for Robotized Inspection. World Conference on NonDestructive Testing, pages 1-8, 2016.

[5] Antonio Bicchi and Giovanni Tonietti. Fast and "soft-arm" tactics IEEE Robotics and Automation Magazine, 11(2):22-33, 2004.

[6] Herman Bruyninckx. OROCOS: design and implementation of a robot control software framework. International Conference on Robotics and Automation (ICRA), 2002.

[7] B. Faverjon and P. Tournassoud. A local based approach for path planning of manipulators with a high number of degrees of freedom. Proceedings. 1987 IEEE International Conference on Robotics and Automation, 4, 1987.

[8] Hans Joachim Ferreau, Christian Kirches, Andreas Potschka, Hans Georg Bock, and Moritz Diehl. qpOASES: a parametric activeset algorithm for quadratic programming. Mathematical Programming Computation, 6(4):327-363, 2014.

[9] Tsutomu Gomi, Hiroshi Hirano, Masahiro Nakajima, and Tokuo Umeda. X-ray digital linear tomosynthesis imaging. Journal of Biomedical Science and Engineering, 2011(June):443-453, 2011.

[10] Elena Gribovskaya, Abderrahmane Kheddar, and Aude Billard. Motion Learning and Adaptive Impedance for Robot Control during Physical Interaction with Humans. International Conference on Robotics and Automation, 2011.

[11] S Haddadin, Alin Albu-Scheffer, and G Hirzinger. Safe physical human-robot interaction: Measurements, analysis \& new insights. International Symposium on Robotics Research (ISRR2007), pages 439-450, 012007.

[12] Alexander Herzog, Nicholas Rotella, Sean Mason, Felix Grimminger, Stefan Schaal, and Ludovic Righetti. Momentum control with hierarchical inverse dynamics on a torque-controlled humanoid. Autonomous Robots, 40(3):473-491, 2016.

[13] Heinzmann; Jochen and Alexander Zelinsky. Quantitative Safety Guarantees for Physical Human Robot Interaction. The International Journal of Robotics Research, 2003.

[14] Alessandro De Luca. Collision Detection and Safe Reaction with the DLR-III Lightweight Manipulator Arm. International Conference on Intelligent Robots and Systems, pages 1623-1630, 2006.

[15] A. Meguenani, V. Paddois, J. Da Silva, A. Hoarau, and P. Bidaud. Energy-based control for safe human-robot physical interactions. In D. Kulic, G. Venture, Y. Nakamura, and O. Khatib, editors, Springer Proceedings in Advanced Robotics - The 2016 International Symposium on Experimental Robotics. International Symposium on Experimental Robotics, 2017.

[16] Anis Meguenani, Vincent Paddois, and Philippe Bidaud. Control of robots sharing their workspace with humans: An energetic approach to safety. IEEE International Conference on Intelligent Robots and Systems, 2015-Decem:4678-4684, 2015.

[17] Sébastien Rubrecht, Vincent Padois, Philippe Bidaud, Michel De Broissia, and Max Da Silva Simoes. Motion safety and constraints compatibility for multibody robots. Autonomous Robots, 32(3):333349, 2012.

[18] Layale Saab, Oscar E Ramos, François Keith, Nicolas Mansard, Philippe Soueres, and Jean-Yves Fourquet. Dynamic whole-body motion generation under rigid contacts and other unilateral constraints. IEEE Transactions on Robotics, 29(2):346-362, 2013.

[19] R. Smits. KDL: Kinematics and Dynamics Library. http://www. orocos.org/kdl.

[20] J. Webster; Stayman and Jeffrey H. Siewerdsen. Task-Based Trajectories in Iteratively Reconstructed Interventional Cone-Beam CT. International Meeting on Fully Three-Dimensional Image Reconstruction in Radiology and Nuclear Medicine, pages 257-260, 2013.

[21] Yoji Yamada, Yasuhiro Hirasawa, Shengyang Huang, Yoji Umetani, and Kazutsugu Suita. Human-robot contact in the safeguarding space. IEEE/ASME Transactions on Mechatronics, 2(4):230-236, 1997. 